\title{
A neonate with tracheobronchial cobblestone mucosa presenting with acute severe hypercapnia
}

\author{
Yong $\mathrm{Liu}^{1}$, $\mathrm{Li} \mathrm{Ly}{ }^{2}$, YuLian $\mathrm{Hu}^{1}$ \\ ${ }^{1}$ Department of Neonatology, Maternal and Child Health Hospital of Hubei Province; ${ }^{2}$ Wuhan Polytechnic, School of \\ Biological Engineering, Wuhan, Hubei, China. E-mail: huyulian1122@outlook.com \\ Received: 18th July 2018, Revised: 14th September 2018, 9th October 2018, Accepted: 16th October 2018
}

\begin{abstract}
SUMMARY: Liu Y, Ly Li, Hu YL. A neonate with tracheobronchial cobblestone mucosa presenting with acute severe hypercapnia. Turk J Pediatr 2019; 61: 971-974.

Here, we describe the case of a neonate suffering from respiratory distress complicated by pulmonary hemorrhage shortly after birth. The neonate experienced repeated dyspnea and acute severe hypercapnia $(160-100 \mathrm{mmHg})$ that began on the 7th day after birth during invasive mechanical ventilation. Rescue high frequency oscillatory ventilation played a key role in maintaining gas exchange during these episodes. Subsequent bronchoscopy revealed tracheobronchial edematous mucosa with a cobblestone pattern, which we believe is the first report of this condition in neonates. Protrusion of the diffuse edematous mucosa led to airway stenosis and respiratory distress. Lesions of the tracheal mucosa should therefore be considered as one potential cause of neonatal respiratory distress.
\end{abstract}

Key words: cobblestone mucosa, acute severe hypercapnia, high frequency oscillation.

Acute severe hypercapnia during invasive ventilation is usually caused by tracheal tube displacement or obstruction, tension pneumothorax or cardiac arrest. Although rare, sedative-induced chest wall stiffness and severe bronchospasm can lead to the same consequences. ${ }^{1,2}$ Here, we report the case of a newborn with tracheobronchial edematous cobblestone mucosa, who presented with respiratory distress complicated by pulmonary hemorrhage soon after birth. The neonate also experienced repeated dyspnea and severe acute hypercapnia during subsequent invasive ventilation.

\section{Case Report}

A full-term male infant, weighing $3.2 \mathrm{~kg}$, and with normal Apgar score, was born by selective cesarean section at 40 weeks plus 1 day of gestation due to his mother's history of cesarean section. Thirty minutes after birth, the baby developed respiratory distress and was supplied with oxygen. Three hours later, he was intubated and a large amount of blood was observed in the endotracheal tube. After the transfusion of red blood cells, he was transported to our hospital for treatment; this occurred within 6 hours of birth.

His mother, a 26-year-old, gravida 2, para 2, had an uneventful pregnancy and received all regular examinations. His three-year-old brother had a history of facial eczema, which may have been associated with an allergy to cow's milk protein. His parents were healthy and without history of allergies and chronic diseases.

Upon admission, the infant's peripheral oxygen saturation was approximately $90 \%$ while he was on intubation and receiving $100 \%$ oxygen via bag ventilation. Intercostal retractions were obvious, and bilateral lung auscultation revealed rales. Mechanical ventilation was duly commenced. Laboratory tests were as follows: hemoglobin $16.5 \mathrm{~g} / \mathrm{dl}$, white blood cell count $16,490 / \mathrm{mm}^{3}$, platelet count $271,000 / \mathrm{mm}^{3}$, and C-reactive protein (CRP) $0.26 \mathrm{mg} / \mathrm{dl}$. Blood cultures were negative. Cerebral and cardiac ultrasound were unremarkable. Chest 
X-ray showed diffuse granular shadows, which had almost disappeared by the second day. On the third day, his general and respiratory status were significantly improved, but we failed to extubate, forcing us to re-intubate. A subsequent chest X-ray revealed bilateral atelectasis with some emphysema.

On the seventh day, the infant suddenly became diaphoretic and cyanotic with severe dyspnea accompanied by some wheezing and rales; breathing sounds were remarkably diminished. Capillary blood gas showed a $\mathrm{pH}$ of 6.8 , and a $\mathrm{pCO}_{2}$ of $160 \mathrm{mmHg}$. Increasing the ventilator settings and replacing the endotracheal tube failed to drive the chest wall. Rescue high-frequency oscillation ventilation (HFOV) was thus commenced using maximum amplitude, a mean airway pressure of $24 \mathrm{~cm}$ $\mathrm{H}_{2} \mathrm{O}$ and a frequency of $6 \mathrm{~Hz}$. Chest wall swing was unapparent at the beginning but slowly became noticeable. The patient's symptoms gradually improved and blood gas returned to normal after two hours. Subsequent X-rays showed that the earlier atelectasis had overexpanded beyond the twelfth rib, and the lung field was clear. Over the following seven days, four similar episodes, in which $\mathrm{pCO}_{2}$ ranged from 100-140 mmHg, occurred soon after tracheal suction, thus requiring rescue HFOV. During the intervals between these episodes, the infant was stable with clear breath sounds. Routine blood cultures did not show any evidence of infection (white cell count was normal) and CRP was within the normal range during this period. Tests for intrauterine infection were also negative. A second cardiac ultrasound did not show any abnormality. We considered that these events were caused by bronchospasms. Intravenous hydrocortisone (2 mg/kg, q6h) and aminophylline (5 mg/kg, q6h) were administered during the second episode; meronpenem and vancomycin were then commenced. Lung computed tomography (CT) showed unevenly inflated lobes and bilateral local bronchial stenosis. Seven days after the first episode, bronchoscopy revealed a tracheal edematous cobblestone mucosa with narrowing of the main bronchi and almost complete occlusion of the lung lobe orifice (Fig. 1). We also carried out bronchoalveolar lavage with budesonide, but found nothing in the bronchoalveolar fluid. No further episodes occurred after this procedure. A week later, a second bronchoscopy revealed significant improvement in the edematous mucosa. Biopsy of the cobblestone mucosa indicated chronic inflammation (Fig. 2). On day 35 after birth, the infant was extubated, and antibiotics and steroids were discontinued. $\mathrm{He}$ was discharged from hospital on the 42nd day.

After one year of follow-up, the infant showed normal development and had seldom suffered from respiratory disease. A second lung CT, performed when the infant was one year old, still showed mild bilateral local bronchial stenosis.

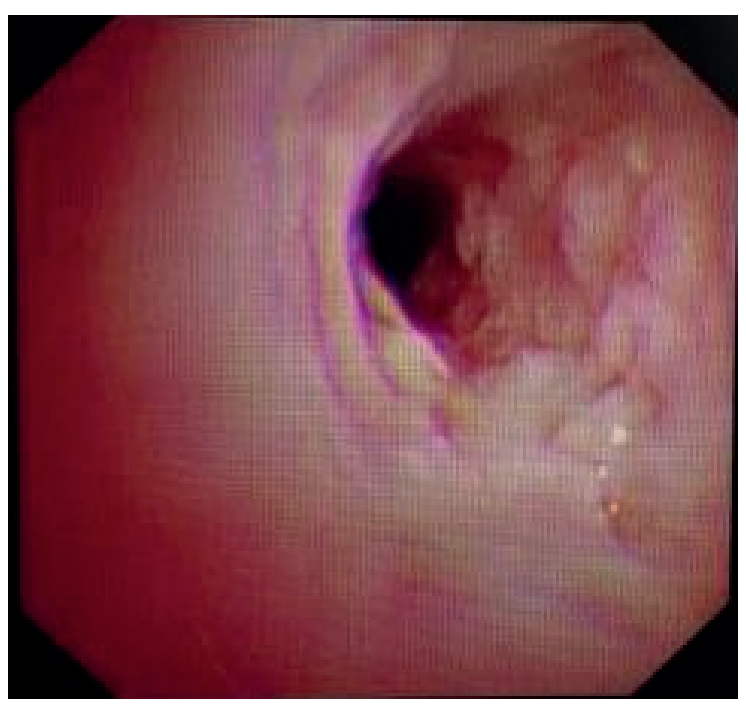

Fig. 1. Bronchoscopy revealed an edematous, glistening, white and nodular bronchial mucosa on an erythematous background.

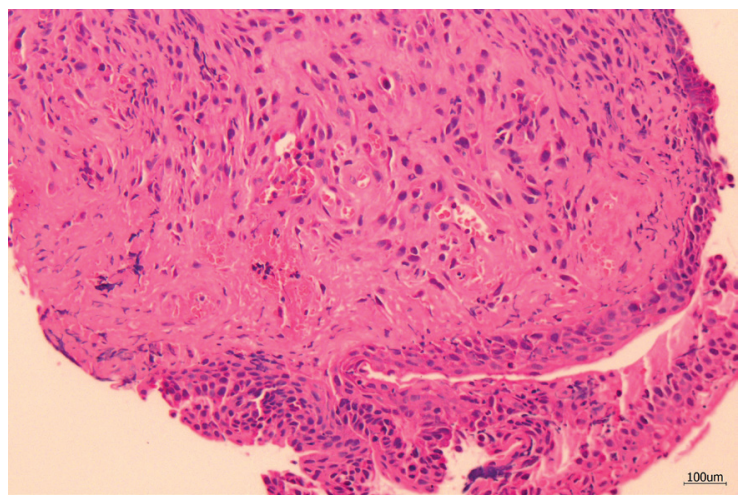

Fig. 2. Cobblestone mucosa in the bronchus indicating an intact epithelial layer, an increased number of fibroblasts and a reduced number of lymphocytes (Sections stained with hematoxylin and eosin, $\times 100)$. 
We obtained informed consent from the patient's family in order to publish this report.

\section{Discussion}

A previous study of preoperative bronchofibroscopy in children reported the incidence of tracheobronchial cobblestone mucosa to be $38.8 \%$; the incidence in children under 3 years of age $(43.5 \%)$ was significantly lower than that of children over 3 years of age $(56.4 \%)$ and cobblestone mucosa was not detected in infants younger than 4 months of age. $^{3}$ The different incidences across different age groups are most likely to be associated with maturity of the immune system. We believe that this is the first report of this type of lesion in neonates. Cobblestone mucosa is usually thought to be an irritant inflammatory response associated with gastroesophageal reflux, respiratory tract infections and aspiration. However, previous research has failed to confirm this assumption. ${ }^{3,4}$ The pathogenesis of tracheobronchial cobblestone mucosa in utero is still unclear, partly because the fetal airway fills with lung fluid. It is difficult for stimulants to enter the airway unless hypoxia causes gasping breathing. In the current case, prenatal monitoring and birth status did not suggest intrauterine hypoxia, and there was no evidence of intrauterine infection. Furthermore, cobblestone mucosal pathology showed chronic inflammation, which also did not support the speculations described above. Respiratory and gastrointestinal epithelia share a common embryonic origin in the primitive foregut, which may account for their structural similarities. Furthermore, there is mounting evidence to show that stimulation of one mucosal compartment can directly and significantly impact upon a distant mucosal site. ${ }^{5}$ Consequently, the tracheal cobblestone mucosa may not only be related to stimuli from the airway, but also from the gut. Exposure of the gut mucosa to antigens in utero is known to induce an immune response ${ }^{6}$; however, we are still unable to provide a satisfactory explanation for the pathogenesis of lesions in the tracheal mucosa in the uterus.

Tracheobronchial cobblestone mucosa does not usually cause respiratory symptoms in children. ${ }^{3,4}$ In adults, edematous cobblestone mucosa has been reported as a manifestation of pulmonary complications in patients with inflammatory bowel disease; in such cases, diffuse edema mucosal protrusions lead to a narrow airway and respiratory distress. ${ }^{7,8}$ This situation is similar to what we observed in our present case.

Respiratory distress and pulmonary hemorrhage can occur shortly after birth, and are usually related to hypoxia and lung damage in utero. However, this particular newborn had no history of intrauterine hypoxia and no obvious abnormality. The fact that the lung shadow evident upon admission disappeared rapidly, along with the failure to extubate, and the atelectasis on the third day, can be explained by the existence of tracheobronchial stenosis. Therefore, the pulmonary hemorrhage may have been related to excessive negative pressure in the alveoli caused by almost three hours of forced breathing to overcome the increased resistance caused by the tracheobronchial stenosis. Due to the tracheobronchial stenosis, a slight reduction in caliber may have increased resistance and aggravated bronchial obstruction in a remarkable manner. The acute severe hypercapnia was characterized by paroxysmality for a duration of 2 hours, which was probably triggered by bronchial muscle contraction rather than aggravated airway edema and secretion retention. Moreover, the pathological manifestation of tracheal cobblestone mucosa involves chronic inflammation, which is also found in children. ${ }^{4}$ Airway inflammation in utero may have increased our patient's reactivity and caused his condition to deteriorate. His episodes ceased after the edematous mucosa had improved, suggesting that such episodes are caused by severe bronchial obstruction due to edematous cobblestone mucosa.

The occurrence of bronchial obstruction is unpredictable and persistent, and medication does not relieve symptoms in a rapid manner. Effective ventilation is very important for maintaining gas exchange during this critical period. Previously, Devaskar et al. ${ }^{2}$ described four neonates who developed seven life-threatening bronchospasms upon conventional ventilation. These neonates had a pCO2 of $>120 \mathrm{mmHg}$ and a $\mathrm{pH}<7.0$. Although very high peak inspiratory pressure 
had been applied, three of these neonates died of respiratory failure. HFOV is not usually recommended for obstructive airway disease, but HFOV, characterized by active expiratory and continuing distension pressure, has been reported to be effective in such cases. ${ }^{9}$ In our present case, the newborn received HFOV. The higher mean airway pressure dilated the narrow bronchus and re-opened the collapsed lung. Maximum amplitude and low frequency ensured that gas exchange could occur. Moreover, our patient experienced five separate episodes but survived without complications.

In conclusion, we describe the first report of tracheobronchial cobblestone mucosa in a neonate, which developed in utero. The pathogenesis of this condition, and the associated implications upon the respiratory system, need to be investigated further. Lesions of the tracheal mucosa can lead to dyspnea, characteristically with obstructive ventilation, and should therefore be considered as one potential cause of neonatal respiratory distress.

\section{Acknowledgements}

The authors would like to thank Mrs. Faith $\mathrm{McNabb}$ for encouraging our research and helping to improve this paper.

\section{REFERENCES}

1. Fahnenstich H, Steffan J, Kau N, Bartmann P. Fentanylinduced chest wall rigidity and laryngospasm in preterm and term infants. Crit Care Med 2000; 28: 836-839.

2. Devaskar UP, Devaskar SU, Keenan WJ. Acute bronchospasm resembling status asthmaticus during the neonatal period. Crit Care Med 1986; 14: 472475 .

3. Dave MH, Gerber A, Bailey M, et al. Prevalence and characteristics of tracheal cobblestoning in children. Pediatr Pulmonol 2015; 50: 995-999.

4. Duval M, Meier J, Asfour F, et al. Association between follicular tracheitis and gastroesophageal reflux. Int J Pediatr Otorhinolaryngol 2016; 82: 8-11.

5. Tulic M K, Piche T, Verhasselt V. Lung-gut crosstalk: evidence, mechanisms and implications for the mucosal inflammatory diseases. Clin Exp Allergy 2016; 46: 519-528.

6. Gerdts V, Snider M, Brownlie R, Babiuk LA, Griebel $\mathrm{PJ}$. Oral DNA vaccination in utero induces mucosal immunity and immune memory in the neonate. J Immunol 2002; 168: 1877-1885.

7. Bhat M, Dawson D. Wheezes, blisters, bumps and runs: multisystem manifestations of a Crohn's disease flare-up. CMAJ 2007; 177: 715-718.

8. Lamblin C, Copin MC, Billaut C, et al. Acute respiratory failure due to tracheobronchial involvement in Crohn's disease. Eur Respir J 1997; 9: 2176-2178

9. Slee-Wijffels FY, van der Vaart KR, Twisk JW, Markhorst DG, Plötz FB. High-frequency oscillatory ventilation in children: a single-center experience of 53 cases. Crit Care 2005; 9: R274-R279. 